\title{
A Critical Commentary on the Practical Application of Resistance Training Studies
}

\begin{abstract}
James Fisher
Objectives: The purpose of this communication is to discuss the need for methodological detail and accuracy in resistance training publications. In doing so the hope is that future research might provide greater clarity, and thus have greater validity serving to (i) advance our physiological understanding of human adaptation, and (ii) provide coaches, trainers, and trainees with methods and results to better accommodate their own practice.

Design and Methods: The sample of literature discussed herein has been considered due to previous exclusion from a larger review article due to a lack of control and/or reporting of independent variables. The present article follows a narrative review format discussing these methodological limitations.

Results: A number of previous publications have failed to control, and/or report with clarity, independent variables such as exercises performed, repetitions, load, contractions, intensity of effort and total training volume both within and between intervention groups.

Conclusions: The results show that a previous lack of control/clarity in resistance training studies can hinder the scientific validity and practical application of any results obtained. By discussing this topic, the hope is to remind researchers, authors, editors, and reviewers of the need for scientific rigour throughout the process of research publication.
\end{abstract}

(Journal of Trainology 2013;2:10-12)

Key words: exercise $\square$ methodology $\square$ strength $\square$ hypertrophy $\square$ training

\section{Introduction}

The practical application of scientific research in the area of resistance training cannot be underestimated. Large organisations such as the American College of Sports Medicine (ACSM) $)^{1}$, and National Strength and Conditioning Association (NSCA) $)^{2,3}$ publish regular recommendations based around peer-reviewed research. Whilst these publications might receive criticism $^{4-6}$ the existence of this debate within an open forum is important for coaches, trainers, and the layperson to consider when following guidelines for their own resistance training prescription.

Whilst many articles consider strength or hypertrophic gains from single exercises a more pragmatic approach might be to consider the effects of 'real-life' training protocols. However the research and publication of such pieces should not be undertaken at the expense of the scientific rigour necessary to accommodate any practical and statistical analysis. Recent publications have suggested differing perspectives on the importance of training variables such as intensity of effort ${ }^{7}$ and volume ${ }^{8}$. However, in context all resistance training variables are crucial to consider, control, and/or measure. As such research should accommodate this clarity to both assist in the growth and progression of comprehensive resistance training research studies, as well as to benefit the individuals for whom this research is completed - coaches, trainers and trainees.

During a recent literature search the author of the present communication was surprised to find a number of published articles which have failed to accurately detail their training protocol; some of which are discussed herein. ${ }^{9-14}$ It seems unnecessary to perform a lengthy systematic review of this area and as such the current piece is a short commentary which highlights the potential limitations of previous publications to assist in the accuracy of future research.

\section{Methodological Ambiguity}

Due to the very nature of this communication it is important to consider the methodological concerns of the research discussed in context of the authors' conclusions. For example Häkkinen and Kallinen ${ }^{9}$ appear to suggest that if a workout is divided into two sessions in a day, equated and compared to a group performing the workout in a single session; that greater hypertrophic gains are possible in the quadriceps. Within their study two groups trained on every second day for 6 weeks, reversing the protocol after 3 weeks (e.g. one group trained $1 \mathrm{x}$ / day for 3 weeks and then divided the workout in to $2 \mathrm{x}$ / day for 3 weeks and vice versa). The authors state that participants performed a squat exercise with a training load of $70-100 \%$ 1RM, for between 1 and 3 repetitions per set, totalling 18-22 contractions per session. Without the clarity of whether this exercise was completed to repetition maximum (RM) or momentary muscular failure (MMF) the knowledge of a range of loads, repetitions, and contractions only serves to suggest a

Received July 30, 2013, accepted August 16, 2013

From the Centre for Health Exercise and Sport Science, Southampton Solent University, East Park Terrace, Southampton, SO14 0YN, UK (J.F.) Communicated by Takashi Abe, PhD

Correspondence to James Fisher, Centre for Health Exercise and Sport Science, Southampton Solent University, East Park Terrace, Southampton, SO14 0YN, UK. Email: James.fisher@Solent.ac.uk 
variance between each participants' training protocol. They continue by stating that an additional quadriceps exercise of either a knee extension or leg press was performed, with a training load between 60 and 80\% 1RM for 5-10 repetitions, totalling 20-40 contractions per exercise. From this detail are we to assume that stimulation and training effect of the quadriceps is equal between an isolated (knee-extension) and a compound (leg press) exercise when training with $20 \%$ greater or lesser load and performing half or double the number of repetitions and contractions? Other research has proposed that the quadriceps have significantly greater involvement in an open-chain knee-extension exercise compared to closed-chain leg press exercise ${ }^{15}$ which suggests any such assumptions would be inaccurate. Häkkinen and Kallinen ${ }^{9}$ continue by stating that the training program included 3 to 4 other exercises each workout for the main muscle groups of the body, however they fail to clarify what these exercises are. From both a scientific and practical aspect we should consider how useful the results from this study are if we don't know exactly what exercises, loads, repetitions, or contractions different participants performed.

A further study considered muscular hypertrophy over a 12 week intervention between middle-aged and elderly participants training using a unilateral (UNIL) or bilateral (BIL) knee extension exercise. ${ }^{10}$ The authors state that both UNIL and BIL groups trained $2 \mathrm{x} /$ week for 12 weeks where the training intervention progressed every 4 weeks beginning with a load of $40-50 \% 1 \mathrm{RM}$ for $10-12$ repetitions for 3-4 sets (weeks 1-4). This progressed to $60-80 \% 1 \mathrm{RM}$ for $6-8$ repetitions, for 3-5 sets (weeks 5-8), and finished with a load of 70-90\% 1RM for 3-6 repetitions for 4-6 sets (weeks 9-12). Again the authors did not clarify that participants trained to $\mathrm{RM}$ or MMF. We might consider the possible variances in training protocol between participants; for example during the first 4-week phase, one person might have performed 12 repetitions for 4 sets at 50\% 1RM, whilst another might have performed 10 repetitions for 3 sets at $40 \% 1 \mathrm{RM}$; if both participants have a hypothetical $1 \mathrm{RM}$ of $100 \mathrm{~kg}$ this equates to total training volume of $2,400 \mathrm{~kg}(12 \times 4 \times 50)$ and $1,200 \mathrm{~kg}(10$ x 3 x 40), respectively. We could further consider the magnitude this might have over the following 4-week phases, including repetitions, loads and sets over a $2 \mathrm{x} /$ week, 12-week period. Each participant performed between a maximum of $70,720 \mathrm{~kg}$ and minimum of $24,960 \mathrm{~kg}$ of total work over the 12 -week period. This shows a considerable disparity between the two training regimes and thus it is worth considering that this intra-group training variation might have been a reason for the lack of between group statistical significance.

In addition other studies have simply been too vague in the training protocol; for example Ahtiainen, et al. ${ }^{11}$ compared 8 male strength athletes (SA) against 8 physically active males (NA) measuring the hypertrophy of the quadriceps muscles following a 21 week intervention. The authors state that "large inter-individual variation was observed in the strength training programs among the present strength athletes" and also that the training program has been presented earlier. ${ }^{12}$
However, upon review of the previous article ${ }^{12}$ the training routine contains so many variations of load, volume, repetition duration, and exercise selection throughout the intervention, that it is simply impossible to know exactly how each participant or group of participants actually trained. Whilst the present author appreciates that this is potentially realistic in its representation of normal variation in a person's training regime, this kind of disparity between participants and groups makes accurate analysis impossible.

Another study, this time considering the effects of concurrent strength and endurance training versus strength training only ${ }^{13}$ details within the methods section that the strength training protocol included:

"four to five exercises for the other main muscle groups of the body (bench press and/or the triceps pushdown and/or lateral pull-down exercise for the upper body; the sit-up exercise for the trunk flexors and/or another exercise for the trunk extensors; and the bilateral/unilateral elbow and/or knee flexion exercise and/or leg adduction/ abduction exercise).... with loads of $50 \%$ to $70 \% 1 R M$... 10-15 repetitions per set....3-4 sets of each exercise".

This lack of scientific clarity continues throughout the rest of the training intervention. Whilst results from this kind of study are intriguing, in reality they likely allow no practical application since no-one can be clear on exactly what protocol to follow.

It might be easy to surmise that since the aforementioned articles $^{9-13}$ are somewhat dated (1991-2003) that this lack of detail no longer occurs in published journal articles. However, more recent evidence exists in a failure to control frequency, load and intensity of effort in a training intervention where the researchers were considering lumbar multifidus cross sectional area. $^{14}$

\section{Conclusion and Future Consideration}

The articles discussed herein, and others, ${ }^{16,17}$ are expected to have undergone ethical approval, an extensive drafting and writing process between multiple authors, and finally a rigorous peer-review/editorial process. It is therefore surprising that such articles reach publication in such esteemed journals when their application is significantly limited by a lack of scientific rigour. We might consider asking for whom resistance training studies are published; are they to satiate the 'publish or perish' attitude, or are they to allow a deeper understanding of exercise physiology, and provide practical guidance to training regimes? With this in mind we urge researchers, authors, editors, and reviewers to have greater vigilance in ensuring any published trial is described with sufficient clarity for exact replication as well as both statistical and practical analysis and application of results.

\section{References}

1. Ratamess NA, Alvar BA, Evetoch TK, et al. American College of Sports Medicine position stand. Progression models in resistance training for healthy adults. Med Sci Sports Exerc 2009; 41: 687-708.

2. Faigenbaum A, Kraemer WJ, Blimkie CJR, et al. Youth resistance training: updated position statement from the national strength and conditioning 
association. J Strength Cond Res 2009; 23(5 Suppl): S60-S79.

3. Kraemer WJ, Szivak TK. Strength training for the warfighter. $J$ Strength Cond Res 2012; 26 Suppl 2: S107-S118.

4. Carpinelli R, Otto RM, Winett RA. A critical analysis of the ACSM position stand on resistance training: insufficient evidence to support recommended training protocols. J Exerc Physiol 2004; 7(3): 1-60.

5. Carpinelli R. Challenging the American College of Sports Medicine 2009 position stand on resistance training. Med Sport 2009; 13: 131-137.

6. Fisher J, Steele J, Bruce-Low S, et al. Evidence-based resistance training recommendations. Med Sport 2011; 15: 147-162.

7. Fisher J, Smith D. Attempting to define "intensity" for muscular performance: is it all wasted effort? Eur J Appl Physiol 2012; 112: 41834185 .

8. Loenneke JP. Skeletal muscle hypertrophy: how important is exercise intensity? J Trainol 2012; 1: 28-31.

9. Häkkinen K, Kallinen M. Distribution of strength training volume into one or two daily sessions and neuromuscular adaptations in female athletes. Electromyogr Clin Neurophysiol 1994; 34: 117-124.

10. Häkkinen K, Kallinen M, Pastinen U-M, et al. Neuromuscular adaptations during bilateral versus unilateral strength training in middle-aged and elderly men and women. Acta Physiol Scand 1996; 158: 77-88.

11. Ahtiainen JP, Pakarinen A, Alen M. Muscle hypertrophy, hormonal adaptations and strength development during strength training in strength- trained and untrained men. Eur J Appl Physiol 2003; 89: 555-563.

12. Häkkinen K, Pakarinen A, Kraemer WJ, et al. Basal concentrations and acute responses of serum hormones and strength development during heavy resistance training in middle-aged and elderly men and women. $J$ Gerontol A Biol Sci Med Sci 2000; 55: B95-B105.

13. Häkkinen $\mathrm{K}$, Alen $\mathrm{M}$, Kraemer WJ, et al. Neuromuscular adaptations during concurrent strength and endurance training versus strength training. Eur J Appl Physiol 2003; 89: 42-52.

14. Willemink MJ, Van Es HW, Helmhout PH, et al. The effects of dynamic isolated lumbar extensor training on lumbar multifidus functional crosssectional area and functional status of patients with chronic nonspecific low back pain. Spine 2012; 37: E1651-E1658.

15. Enocson AG, Berg HE, Vargas R, et al. Signal intensity of MR-images of thigh muscles following acute open- and closed chain kinetic knee extensor exercise - index of muscle use. Eur J Appl Physiol 2005; 94: 357 363.

16. Häkkinen K, Kallinen M, Komi PV, et al. Neuromuscular adaptations during short-term "normal" and reduced training periods in strength athletes. Electromyogr Clin Neurophysiol 1991; 31: 35-42.

17. Häkkinen K, Pakarinen A, Kallinen M. Neuromuscular adaptations and serum hormones in women during short-term intensive strength training. Eur J Appl Physiol 1992; 64: 106-111. 